\title{
Prevalence of underweight in the Roma population from a rural area in the south part of Romania- Călăraşi county
}

\author{
Emilia Rusu ${ }^{1}$, Georgiana Enache ${ }^{2,3}$, Alexandra Alexescu ${ }^{4}$, Florin Rusu ${ }^{5}$, Andrada Cosoreanu ${ }^{6}$, \\ Catalina Bejinariu' ${ }^{6}$, Mariana Jinga ${ }^{1,5}$ and Gabriela Radulian ${ }^{7,1}$ \\ "Carol Davila" University of Medicine and Pharmacy, Bucharest, Romania, \\ ${ }^{2}$ County Emergency Hospital "Dr Pompei Samarian, Bucharest, Romania, \\ ${ }^{3}$ Carol Davila" University of Medicine and Pharmacy, Bucharest, Romania, \\ 4"Carol Davila" University of Medicine and Pharmacy", Bucharest, Romania, \\ "Carol Davila" Clinical Emergency Military Hospital, Bucharest, Romania, \\ "Nicolae Malaxa" Clinical Hospital, Bucharest, Romania and \\ 7“Prof. N.C. Paulescu” National Institute of Diabetes, Nutrition and Metabolic Diseases, Bucharest, Romania
}

\begin{abstract}
Background and aim: The Roma population is one of the ethnic minorities with a long history of malnutrition. The aim of this study was to evaluate the prevalence of underweight in Roma population living in rural settlements from Călăraşi County in the south part of Romania.

Methods: This cross-sectional, epidemiological, non-interventional study was conducted from March 2014 to May 2017 in several villages from Calarasi County. We analized the data from 978 people: 660 Roma (457 females/203 males), and 318 Romanian Caucasians (213 females/105 males) aged between 18 and 88 yr. Body mass index (BMI) was classified using the definitions of World Health Organization and a BMI $<18.5 \mathrm{~kg} / \mathrm{m}^{2}$ was defined as underweight.
\end{abstract}

Results: In Roma population group, the prevalence of underweight, normal weight, overweight and obesity was $4.5 \%(\mathrm{n}=30), 26.5 \%$ $(\mathrm{n}=175), 25 \%(\mathrm{n}=165)$ and $43.9 \%(\mathrm{n}=290)$ respectively. The prevalence of underweight was higher in Roma population compared to Romanian Caucasian, with a significantly higher prevalence in young age groups, 6.9\% in 18-29-year age group and $8.2 \%$ in $30-39$-year age group as well as in the age group of over 70 years $(7.5 \%)$.

Among the Roma population, in multivariate adjustment for all covariates in a backward stepwise elimination procedure, significant predictors of underweight were a current smoking and lower socio-educational level (under eight classes).

Conclusions: Our results confirm and reinforce the need to implement prevention programs in high-risk populations such as the Roma population, due to the double burden of malnutrition, low access to the health system, low socio-economic level, limited access to education lack of medical education and preventive healthcare.

\section{Conflict of Interest}

There is no conflict of interest. 